BULL. AUSTRAL. MATH. SOC.

VOL. $25(1982), 473-474$.

\title{
CYCLOTOMY AND DESIGNS
}

\author{
Deborah J, Street
}

The central theme of the work in this thesis is the construction, by cyclotomic methods, of various kinds of designs. Chapter 1 gives the basic background. The next four chapters discuss four different kinds of designs constructed using the theory of cyclotomy. The final two chapters generalise some of these designs using other methods of construction.

In Chapter 2 the relationship between generalised Hadamard matrices, orthogonal arrays and $F$-squares is investigated. This gives some reasonably large sets of mutually orthogonal $F$-squares; that is, sets which contain close to the maximum number possible.

In Chapter 3 one generalisation of weighing matrices is considered. This leads to some constructions for Bhaskar Rao designs and for partially balanced incomplete block designs, all based on a family of supplementary difference sets obtained by generalising results of Eimma Lehmer. These difference sets are used extensively in Chapter 5 in the construction of Graeco-Latin and nested row and column designs. These designs are for two sets of treatments, with the arrangement of the treatment sets relative both to each other and to the blocks being of importance.

In Chapter 4 balanced incomplete block designs, in which ordered pairs of elements occur equally often in blocks, are considered. It is shown that the necessary conditions are sufficient for $k=4$ and $k=5$, with the exception of the design with $v=15, k=5, \lambda^{*}=1$.

The final two chapters are of a rather different nature. The results in Chapter 6 are an extension of those in Chapter 2 but are obtained by

Received 22 February 1982. Thesis submitted to the University of Sydney, August 1981. Degree approved February 1982. Supervisor: Dr Jennifer Seberry. 
group-theoretic methods; those in Chapter 7 extend those in Chapter 3 by direct search.

Department of Mathematics,

University of Queensland,

St Lucia,

Queensland 4067,

Australia. 